\title{
Numerical code of the neutrino-transfer in three dimensions for core-collapse supernovae
}

\author{
Kohsuke Sumiyoshi ${ }^{1}$ and Shoichi Yamada ${ }^{2}$ \\ ${ }^{1}$ Numazu College of Technology, Ooka 3600, Numazu, Shizuoka 410-8501, Japan \\ email: sumi@numazu-ct.ac.jp \\ ${ }^{2}$ Science and Engineering \& Advanced Research Institute for Science and Engineering, \\ Waseda University, Okubo, 3-4-1, Shinjuku, Tokyo 169-8555, Japan \\ email: shoichi@heap.phys.waseda.ac.jp
}

\begin{abstract}
We develop a new numerical code of the multi-energy and multi-angle neutrinoradiation transfer in three dimensions (3D) for core-collapse supernovae. Our 3D code to solve the Boltzmann equations is based on the discretized-ordinate $\left(\mathrm{S}_{N}\right)$ method with a fully implicit differencing for time advance. A basic set of neutrino reactions is implemented in the collision terms together with a realistic equation of state. By following the time evolution of neutrino distributions in six dimensions (3 spatial and 3 momentum-space) by the 3D Boltzmann solver, we study the 3D feature of neutrino transfer for given background models of supernova cores in order to understand the explosion mechanism through neutrino heating in multi dimensions.
\end{abstract}

Keywords. methods: numerical, neutrinos, radiative transfer, stars: massive, stars: neutron, supernovae: general

\section{Challenges to compute the neutrino transfer in supernovae}

Computation of the neutrino-radiation transfer together with hydrodynamics is mandatory for the study of gravitational collapse of massive stars. The neutrino heating mechanism in non-spherical configurations induced by hydrodynamical instabilities is the essential key to clarify the elusive mechanism of supernova explosion. Simplifications of the neutrino transfer by dropping energy or angle dependence are not reliable in principle since neutrino energy- and angle-dependent interactions determine the transfer of energy in the supernova dynamics.

To pin down the crucial part of the explosion mechanism, full calculations of the neutrino-radiation hydrodynamics in $3 \mathrm{D}$ without invoking approximations of neutrino transfer are required. In two dimensions, the approximate treatments of neutrino transfer have been adopted in most of the state-of-the-art calculations (Kotake (2012)). Only recently the Boltzmann equations in axial symmetry were directly solved by Ott et al. (2008). The 3D numerical simulations with an approximate neutrino transfer have been reported more recently (Takiwaki, Kotake \& Suwa (2012)) in the ray-by-ray approach.

\section{Applications of the new 3D Boltzmann solver}

Rapid growth of supercomputing power enables us to compute the neutrino transfer in 3D (Sumiyoshi \& Yamada (2012)). It is a challenging task to describe the time evolution of neutrino distributions in six dimensions (three spatial coordinates with one energy and two angles). We have developed a numerical code to solve the Boltzmann equations for multi-energy and multi-angle group in $3 \mathrm{D}$ spatial coordinates. We solve the time 

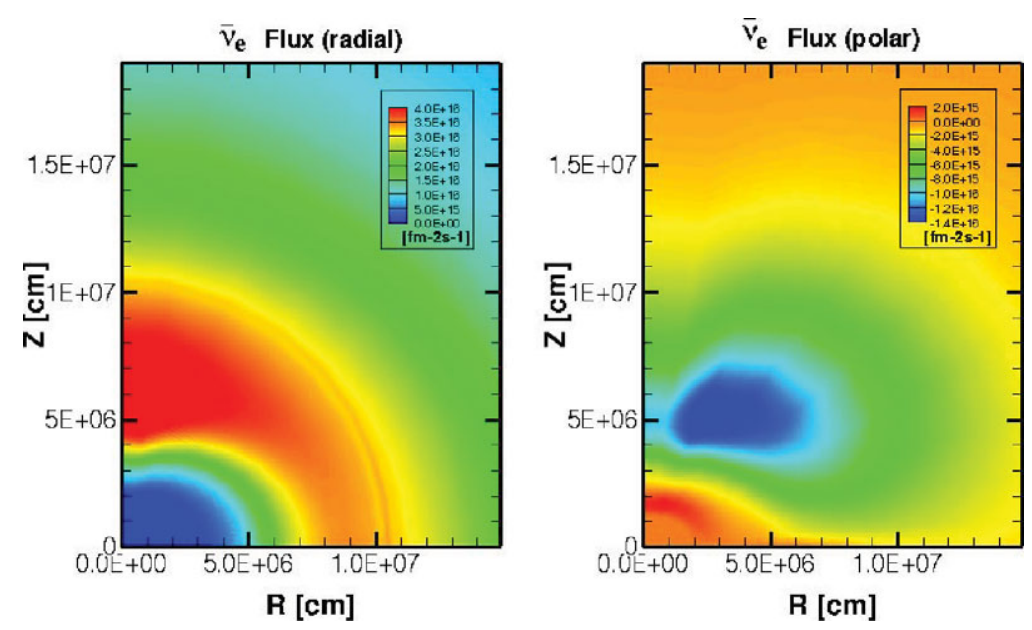

Figure 1. Radial (left) and polar (right) components of the fluxes of electron-type anti-neutrinos in the 2D deformed supernova core on a meridian slice.

evolution of neutrino distributions by the discrete-ordinate $\left(\mathrm{S}_{n}\right)$ method with a fully implicit differencing for time advance. The basic set of neutrino reactions (including pair processes) for the neutrinos of three species $\left(\nu_{e}, \bar{\nu}_{e}, \nu_{\mu / \tau}\right)$ is implemented with a set of EOS table (Sumiyoshi et al. (2005), Shen et al. (2011)).

The 3D Boltzmann solver is applied to examine the neutrino transfer for given background profiles in 2D/3D from Takiwaki, Kotake \& Suwa (2012) and Sekiguchi \& Shibata (2011). In Figure 1, we show an exemplar model of axially deformed supernova cores after the bounce. Due to the oblate shape of the proto-neutron star at center, the radial flux is enhanced near the polar direction. The contribution of the polar flux ( $\theta$-direction) is substantial between the pole and the equatorial plane. We note that this feature can be properly described by the 3D Boltzmann solver, being different from the ray-by-ray methods, in which only the radial flux can be described. Developments of the numerical code for the 3D neutrino-radiation hydrodynamics are in progress in order to explore the 3D supernova dynamics with the exact solution of neutrino transfer.

This work is based on the collaboration with H. Nagakura, S. Furusawa, H. Matsufuru, A. Imakura, T. Sakurai, Y. Sekiguchi, T. Takiwaki and K. Kotake. The numerical computations were performed on the supercomputers at YITP, KEK, RCNP, UT, Japan. This work is supported by the Grant-in-Aid for Scientific Research (Nos. 19104006, 20105004, 20105005, 21540281, 22540296) and the HPCI Strategic Program by MEXT, Japan.

\section{References}

Kotake, K. 2012, Comptes Rendus Physique in press; arXiv:1110.5107

Ott, C. D., Burrows, A., Dessart, L., \& Livne, E. 2008, ApJ, 685, 1069

Sekiguchi Y. \& Shibata M. 2011, ApJ, 737, 6

Shen, H., Toki, H., Oyamatsu, K., \& Sumiyoshi, K. 2011, ApJS, 197, 20

Sumiyoshi, K., Yamada, S., Suzuki, H., Shen, H., Chiba, S., \& Toki, H. 2005, ApJ, 629, 922

Sumiyoshi, K. \& Yamada, S. 2012, ApJS, 199, 17

Takiwaki, T., Kotake, K., \& Suwa, Y. 2012, ApJ, 749, 98 